\title{
The facing bias in biological motion perception: Effects of stimulus gender and observer sex
}

\author{
BeN SCHOUTEN \\ University of Leuven, Leuven, Belgium \\ Nikolaus F. Troje \\ Queen's University, Kingston, Ontario, Canada \\ ANNA BROOKS AND RICK VAN DER ZWAN \\ Southern Cross University, Coffs Harbour, New South Wales, Australia \\ AND \\ KARL VERfaillie \\ University of Leuven, Leuven, Belgium
}

\begin{abstract}
Under orthographic projection, biological motion point-light walkers offer no cues to the order of the dots in depth: Views from the front and from the back result in the very same stimulus. Yet observers show a bias toward seeing a walker facing the viewer (Vanrie, Dekeyser, \& Verfaillie, 2004). Recently, we reported that this facing bias strongly depends on the gender of the walker (Brooks et al., 2008). The goal of the present study was, first, to examine the robustness of the effect by testing a much larger subject sample and, second, to investigate whether the effect depends on observer sex. Despite the fact that we found a significant effect of figure gender, we clearly failed to replicate the strong effect observed in the original study. We did, however, observe a significant interaction between figure gender and observer sex.
\end{abstract}

In 1973, Gunnar Johansson demonstrated that despite degradation of visual information to just a few dots (point lights) attached to the major joints of an otherwise invisible actor, observers are able to effortlessly recognize human actions. Since then, it has been reported that humans are able to extract a variety of behaviorally relevant characteristics, including the type of action (Dittrich, 1993; Vanrie \& Verfaillie, 2004), the identity of the actor (Cutting \& Kozlowski, 1977; Loula, Prasad, Harber, \& Shiffrar, 2005), and the actor's gender (Kozlowski \& Cutting, 1977; Pollick, Kay, Heim, \& Stringer, 2005; Troje, 2002), emotional state (Dittrich, Troscianko, Lea, \& Morgan, 1996; Montepare, Goldstein, \& Clausen, 1987), and age (Montepare \& Zebrowitz-McArthur, 1988). Only more recently, however, has the question of how observers interpret the in-depth orientation of point-light figures systematically been addressed (Jackson, Cummins, \& Brady, 2008; Vanrie, Dekeyser, \& Verfaillie, 2004; Vanrie \& Verfaillie, 2006, 2008).

A point-light walker without explicit depth cues constitutes a perfectly ambiguous stimulus. Any nonlateral view of the walker could in principle be the result of a 2-D projection of two different veridical 3-D orientations. For instance, the orthographic projection of a 3-D walker facing the viewer in frontal view is identical to the projec- tion of a walker who faces away from the viewer. Both interpretations are equally plausible in principle. Yet observers exhibit a perceptual bias to interpret the figure as being oriented toward the viewer (Vanrie et al., 2004). An orthographically projected frontal/back view pointlight walker generally elicits more than $80 \%$ "facing the viewer" (FTV) responses.

To date, the causal factors of this facing bias are unclear. As argued by Vanrie et al. (2004), there is in principle no reason to assume that observers are confronted visually more often with frontal views than with back views of their conspecifics. Consequently, an explanation based on familiarity seems inadequate. An account based on social and biological relevance offers an alternative possibility. One could argue that, in most cases, another person facing the observer probably is socially more relevant than a person facing away. From this perspective, the perceptual system might take into account the potential cost of misinterpreting the actions and intentions of others: Expecting someone to approach who is actually retreating might be less costly than misinterpreting an approach for a retreat. Such an account is consistent with existing explanations of the well-documented perceptual bias for rising tones, which signal a looming object or entity in the auditory modality (Bach et al., 2008; Neuhoff, 1998), or of the pref-

B. Schouten, ben.schouten@psy.kuleuven.be 
erential responsiveness to visual and audiovisual looming signals (Maier, Neuhoff, Logothetis, \& Ghazanfar, 2004; Schiff, Caviness, \& Gibson, 1962).

Following this line of reasoning, in an initial study we (Brooks et al., 2008) recently investigated the effect of the perceived gender of an ambiguous point-light figure on its perceived in-depth orientation. In the study of Vanrie et al. (2004), only male walkers were shown. However, if social and biological relevance indeed affect perceptual in-depth organization then, we reasoned, the cost of misinterpreting actions could be different for the perception of male conspecifics than for the perception of female conspecifics. For instance, an approaching male might be more threatening than an approaching female (see Gunns, Johnston, \& Hudson, 2002, for related research on the opposite effect - namely, how movement information can specify how vulnerable another person seems to be). Therefore, male and female ambiguous point-light figures might elicit different degrees of the facing bias. Using depth-ambiguous point-light figures in frontal/ back view, and varying gender on a continuum from extremely male to extremely female (see Troje, 2002), we collected both gender ratings and in-depth orientation ratings. A strong correlation between perceived gender and perceived orientation of ambiguous point-light walkers was observed. Consistent with the facing bias found by Vanrie et al., figures perceived as male were generally perceived as facing the viewer. The remarkable finding was that the more the point-light figures were perceived as female, the more the figures were perceived as facing away from the viewer.

The purpose of the present study was twofold. First, the number of participants tested in our initial study was relatively small $(n=5)$. We examined the robustness of the effect by testing a larger subject sample. Second, in our initial study, no observer sex differences were found. In fact, since it was not the goal of that study, the sample size was too small ( 2 male and 3 female participants) to reliably test for an effect of observer sex. To this end, 20 female and 20 male participants completed the same task as in Experiment 1 of Brooks et al. (2008).

\section{METHOD}

\section{Participants}

Forty students ( 20 female, 20 male) from the University of Leuven participated for course credit. All observers had normal or correctedto-normal vision and were naive to the purpose of the experiment.

\section{Stimuli and Apparatus}

The stimuli and procedure were designed to be as similar as possible to those of Experiment 1 of Brooks et al. (2008). Each pointlight walker was presented in frontal view in the center of the screen (subtending a size of $8^{\circ}$ of visual angle in height) and each consisted of 15 black dots (radius $=15$ arc min of visual angle) on a gray background. The gender of the point-light figures was gradually manipulated from extremely female to extremely male. As in our initial study, the two-dimensional positions of the point lights were derived from the gender continuum described in detail by Troje (2002). Seven sets of two-dimensional positions were chosen to be equally spaced between extremely female and extremely male
( $z$ scores of $-6,-4,-2,0,2,4,6 S D$, based on the distribution of 100 individual walkers spanning the walker space; for details, see Troje, 2008). Each stimulus presentation consisted of 3 walking cycles ( $1.5 \mathrm{sec} /$ cycle), each comprising 2 steps (128 frames/cycle at 85 frames/sec). The start position of the animation cycle was randomized across trials.

\section{Procedure}

All observers were tested individually. They were seated in a dimly lit and sound-attenuated room in front of a 21-in. CRT monitor. Viewing distance was $57 \mathrm{~cm}$. The experiment consisted of two phases: a gender judgment phase and a facing judgment phase. To control for the effect of order, the order of the gender judgment task and the facing judgment task was counterbalanced across observers. Before the start of the experiment, observers were informed only about the contents of the first task (gender or facing judgment) and were unaware about the contents of the second task (facing or gender judgment, respectively). Observers were instructed to indicate on each trial by a keypress whether they perceived the presented stimulus as facing toward them (FTV) or facing away (FA) from them (arrow down for FTV, arrow up for FA) in the facing judgment phase, or whether the presented stimulus was perceived as female or male (left arrow, right arrow) in the gender judgment phase. They were instructed to respond according to their own subjective experience, and it was stressed that an equal distribution of both response alternatives was not necessary. After instructions were given, the observers completed a practice block (random selection of all possible conditions) in which they were familiarized with the stimuli and the task. Then we checked again whether they had understood the task, and the experiment commenced. Within each phase, trials were randomized and divided in blocks of 35 , between which participants could take a break. In total, each participant completed 420 trials ( 7 genders $* 2$ phases $*$ 30 repetitions).

\section{RESULTS}

In Figure 1A, we have plotted the proportions of FTV responses as a function of figure gender, averaged across all observers (black), female observers only (white), and male observers only (gray). For comparison, we also show proportions of FTV responses from the initial study (light gray squares). Figure 1A clearly shows that the pattern of proportions of FTV responses as a function of figure gender in the present experiment (black circles) is highly different from the pattern reported in Brooks et al. (2008; light gray squares). In that initial study, we found that the facing bias was strongly modified by figure gender. Although we observed close to $100 \%$ FTV responses for walkers that appeared extremely male, this percentage dropped to $10 \%$ for extremely female walkers.

The present data look quite different. Although the overall facing bias was about the same as in the initial study ( $70 \%$ of FTV responses in the present study and $68 \%$ in the initial study), the effect of figure gender was much weaker. For gender-neutral walkers and within the male part of the stimulus range, the facing bias was about constant, with values around $75 \%$ FTV responses. Only in the strongly female part of the range ( $z$ scores smaller than -2 ) did these values drop and reach a value of $52 \%$ for the most extreme female walker at $-6 S D$. Even though the effect of figure gender was weaker than reported in our initial study, it is still highly significant [two-way re- 


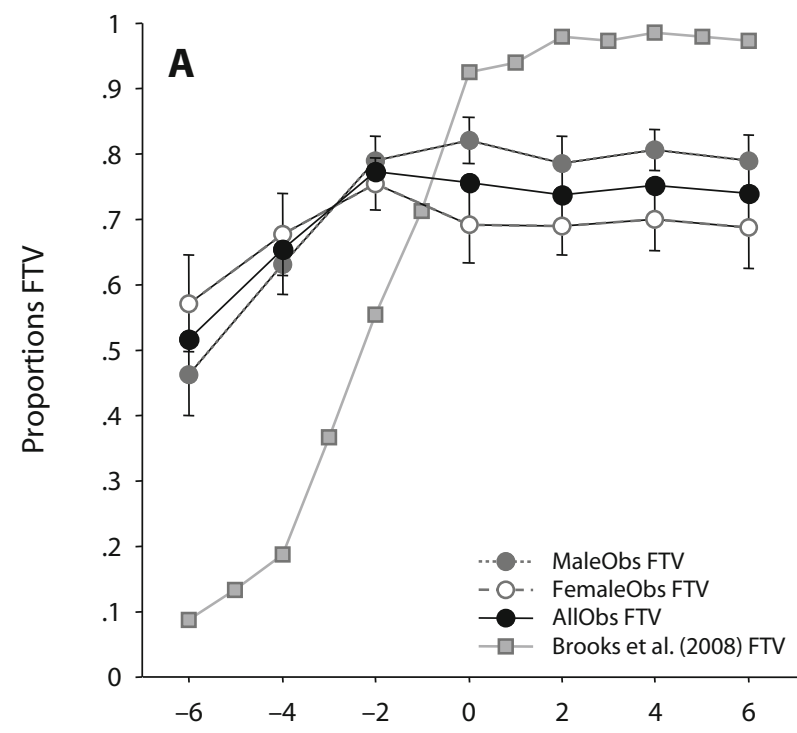

Figure Gender

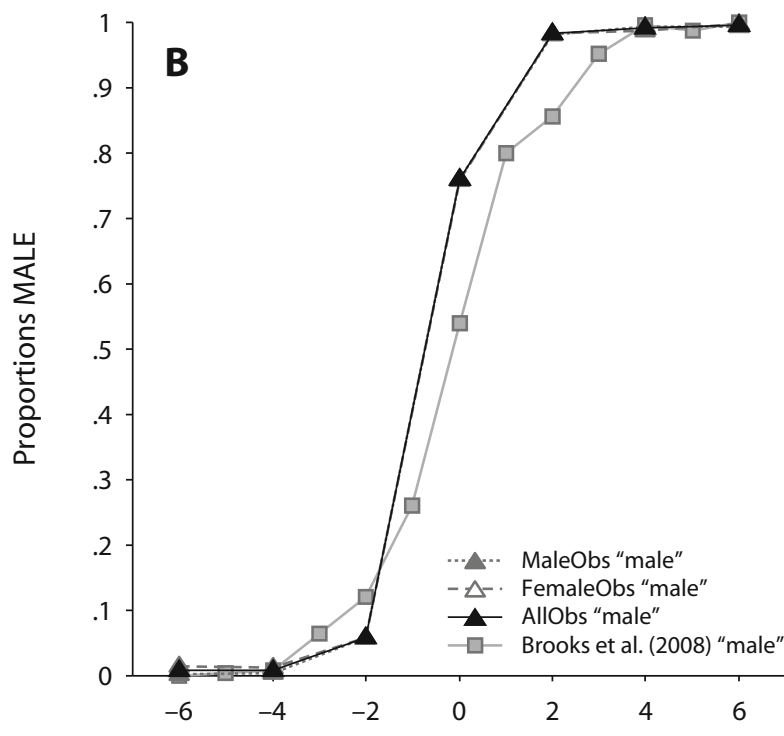

Figure Gender

Figure 1. Mean proportions of FTV (A) and "male" (B) responses as a function of figure gender for all observers (black), female observers (white), and male observers (gray) in the present experiment. Note that "male" responses from male and females observers were so similar that the three symbols overlap and cannot be distinguished in panel B. Data from Experiment 1 in Brooks et al. (2008) are also depicted (light gray squares). Error bars indicate $\pm 1 S E$ around the mean proportions of FTV responses for male and female observers.

peated measures ANOVA on probit transformed proportions; $F(6,228)=7.10, p<.001]$. The order of the phases (gender/facing vs. facing/gender) did not have an effect on the facing bias $(F<1)$, nor did it interact with the figure gender effect $(F<1)$.

Figure 1A also depicts the effect of observer sex on the relation between figure gender and facing bias. Although observer sex itself did not show a main effect on facing bias, we found a significant interaction between figure gender and observer sex $[F(6,228)=2.24, p<.5]$. Figure gender seems to have a stronger effect on male observers than on female observers. In comparison with female observers, male observers showed a generally higher facing bias for male walkers.

Figure 1B shows the proportions of "male" responses as a function of figure gender, averaged across all observers (black), female observers only (white), and male observers only (gray). For comparison, the proportions of "male" responses from the initial study are also depicted (light gray squares). The data show that the walkers at the two ends of the figure gender spectrum were unanimously rated either female or male by every single observer and for any single trial. Ambiguous ratings were only found in the range between -2 and $+2 S D$. In contrast to the data obtained in the initial study $(M=$ $-0.0970,95 \%$ confidence interval $=-0.27$ to 0.08 ; proportions fitted with a cumulative Gaussian; confidence intervals based on 10,000 Monte Carlo simulations; see Wichmann \& Hill, 2001a, 2001b), the curve was shifted a little to the left $(M=-0.61,95 \%$ confidence interval $=$ -0.67 to -0.55$)$, indicating a bias toward responding "male" more often than "female." This "male bias" has been described before (Troje, Sadr, Geyer, \& Nakayama, 2006; Troje \& Szabo, 2006; van der Zwan et al., 2009). Recovering it in the present data makes us confident that they are more representative than the data obtained from the smaller number of participants used in the initial study (Brooks et al., 2008).

Figure 1B also shows the proportions of "male" responses as a function of figure gender for female (white triangles) and male (gray triangles) observers, separately. However, the two curves are so similar that they cannot even be distinguished in Figure 1B.

\section{DISCUSSION}

In the present experiment, we did not replicate the results of Brooks et al. (2008). Indeed, the pattern of proportions of FTV responses as a function of figure gender observed here differed quite strongly from the pattern observed previously. We did observe an effect of figure gender, but it was much weaker than in the initial study. Interestingly, we found an interaction between stimulus gender and observer sex. Additionally, in accordance with previous research, in the present experiment we observed two types of biases. First, we confirmed the facing bias previously reported in studies on the in-depth perception of point-light figures (Schouten \& Verfaillie, 2010; Vanrie et al., 2004; Vanrie \& Verfaillie, 2006, 2008). Second, we observed a "male bias" similar to findings in other studies (Troje et al., 2006; Troje \& Szabo, 2006; van der Zwan et al., 2009) that have re- 
ported gender ratings on the point-light walkers along the gender continuum created by Troje (2002; see also Troje, 2008).

Although the present study constitutes an important rectification of Brooks et al. (2008), several questions remain. First, it remains puzzling why the data in the present experiment are so different from the data reported previously. In the present experiment, 40 observers who were naive to the goal of the experiment participated, as opposed to only 5 observers in our initial study, 4 of whom were experienced psychophysical observers. It could be the case that experienced observers in the initial study attended to different properties of the stimuli than did naive observers. Alternatively, experimenter effects may have influenced the observers' responses in the initial study. We believe that the present data give a more refined and reliable reflection of the perceived in-depth orientation of the point-light figures along the gender continuum.

Next, the question remains why the perceived in-depth orientation depends to some degree on the figure gender and why this dependency is modulated by the sex of the observer. In line with what was suggested in Brooks et al. (2008), one explanation for the effect of figure gender could be that if the facing bias reflects an adaptation to the higher cost of misinterpreting an approaching person, misinterpreting the approach of a female figure might be less of a risk than misjudging the approach of a more dangerous male figure. This view would also provide a hypothesis about the observed interaction between stimulus gender and observer sex: Males are probably more likely to become involved in fights than females. Therefore, the difference in the costs involved with the two kinds of possible errors that an observer can make might be higher for men than for women.

An alternative to this evolutionarily motivated explanation is that simple stimulus properties might be involved that trigger a particular in-depth perception independently of the perceived gender. That is, particular configural or kinematic information correlated with stimulus gender might be responsible for the figure gender effect. For instance, some of the geometric features that distinguish male and female point-light walkers may be misinterpreted as perspective distortions (see Schouten \& Verfaillie, 2010). In that case, the interaction between figure gender and observer sex could be the result of the fact that, especially when it concerns figures that signal a particular gender (Hewig, Trippe, Hecht, Straube, \& Miltner, 2008; Johnson \& Tassinary, 2005), male observers attend to different parts of the figure than do female observers. Future research should investigate whether or not it is possible to identify stimulus properties that trigger a particular in-depth orientation perception in depth-ambiguous point-light figures.

\section{AUTHOR NOTE}

This research was supported by the Scientific Research FundFlanders, FWO G.0621.07 to K.V., and grants from NSERC and CIFAR to N.F.T. Correspondence concerning this article should be addressed to B. Schouten, Tiensestraat 102, 3000 Leuven, Belgium (e-mail: ben .schouten@psy.kuleuven.be).

\section{REFERENCES}

Bach, D. R., Schachinger, H., Neuhoff, J. G., Esposito, F., Di Salle, F., Lehmann, C., ET Al. (2008). Rising sound intensity: An intrinsic warning cue activating the amygdala. Cerebral Cortex, 18, 145-150. doi:10.1093/cercor/bhm040

Brooks, A., Schouten, B., Troje, N. F., Verfaillie, K., Blanke, O., \& VAN DER ZWAN, R. (2008). Correlated changes in perceptions of the gender and orientation of ambiguous biological motion figures. Current Biology, 18, 728-729. doi:10.1016/j.cub.2008.06.054

Cutting, J. E., \& Kozlowski, L. T. (1977). Recognizing friends by their walk: Gait perception without familiarity cues. Bulletin of the Psychonomic Society, 9, 353-356.

DitTRICH, W. H. (1993). Action categories and the perception of biological motion. Perception, 22, 15-22. doi:10.1068/p220015

Dittrich, W. H., Troscianko, T., Lea, S. E. G., \& Morgan, D. (1996). Perception of emotion from dynamic point-light displays represented in dance. Perception, 25, 727-738. doi:10.1068/p250727

Gunns, R. E., Johnston, L., \& Hudson, S. M. (2002). Victim selection and kinematics: A point-light investigation of vulnerability to attack. Journal of Nonverbal Behavior, 26, 129-158. doi:10.1023/A:1020744915533

Hewig, J., Trippe, R. H., Hecht, H., Straube, T., \& Miltner, W. H. R. (2008). Gender differences for specific body regions when looking at men and women. Journal of Nonverbal Behavior, 32, 67-78. doi:10.1007/s10919-007-0043-5

JaCkson, S., Cummins, F., \& Brady, N. (2008). Rapid perceptual switching of a reversible biological figure. PLOS ONE, 3, e3982. doi:10.1371/journal.pone.0003982

Johansson, G. (1973). Visual perception of biological motion and a model for its analysis. Perception \& Psychophysics, 14, 201-211.

Johnson, K. L., \& Tassinary, L. G. (2005). Perceiving sex directly and indirectly: Meaning in motion and morphology. Psychological Science, 16, 890-897. doi:10.1111/j.1467-9280.2005.01633.x

KozlowsKi, L. T., \& CutTing, J. E. (1977). Recognizing the sex of a walker from a dynamic point-light display. Perception \& Psychophysics, 21, 575-580.

Loula, F., Prasad, S., Harber, K., \& Shiffrar, M. (2005). Recognizing people from their movement. Journal of Experimental Psychology: Human Perception \& Performance, 31, 210-220. doi:10.1037/0096 $-1523.31 .1 .210$

Maier, J. X., Neuhoff, J. G., Logothetis, N. K., \& GhaZanfar, A. A. (2004). Multisensory integration of looming signals by rhesus monkeys. Neuron, 43, 177-181. doi:10.1016/j.neuron.2004.06.027

Montepare, J. M., Goldstein, S. B., \& Clausen, A. (1987). The identification of emotions from gait information. Journal of Nonverbal Behavior, 11, 33-42. doi:10.1007/BF00999605

Montepare, J. M., \& ZebrowitZ-McArthur, L. (1988). Impressions of people created by age-related qualities of their gaits. Journal of Personality \& Social Psychology, 55, 547-556. doi:10.1037/0022-3514 .55.4.547

Neunoff, J. G. (1998). Perceptual bias for rising tones. Nature, 395, 123-124. doi: $10.1038 / 25862$

Pollick, F. E., Kay, J. W., HeIm, K., \& Stringer, R. (2005). Gender recognition from point-light walkers. Journal of Experimental Psychology: Human Perception \& Performance, 31, 1247-1265. doi:10.1037/ 0096-1523.31.6.1247

Schiff, W., Caviness, J. A., \& Gibson, J. J. (1962). Persistent fear responses in rhesus monkeys to the optical stimulus of "looming." Science, 136, 982-983. doi:10.1126/science.136.3520.982

Schouten, B., \& Verfaillie, K. (2010). Determining the point of subjective ambiguity of ambiguous biological-motion figures with perspective cues. Behavior Research Methods, 42, 161-167. doi:10.3758/ BRM.42.1.161

TroJE, N. F. (2002). Decomposing biological motion: A framework for analyses and synthesis of human gait patterns. Journal of Vision, $\mathbf{2}$, 371-387. doi:10.1167/2.5.2

TRoje, N. F. (2008). Retrieving information from human movement patterns. In T. F. Shipley \& J. M. Zacks (Eds.), Understanding events: 
How humans see, represent, and act on events (pp. 308-334). Oxford: Oxford University Press.

Troje, N. F., Sadr, J., Geyer, H., \& Nakayama, K. (2006). Adaptation aftereffects in the perception of gender from biological motion. Journal of Vision, 6, 850-857. doi:10.1167/6.8.7

Troje, N. F., \& Szabo, S. (2006, May). Why is the average walker male? Poster presented at the Vision Sciences Society meeting, Sarasota, FL. van der Zwan, R., Machatch, C., Kozlowski, D., Troje, N. F., Blanke, O., \& Brooks, A. (2009). Gender bending: Auditory cues affect visual judgements of gender in biological motion displays. Experimental Brain Research, 198, 373-382. doi:10.1007/s00221-009 $-1800-\mathrm{y}$

Vanrie, J., Dekeyser, M., \& Verfaillie, K. (2004). Bistability and biasing effects in the perception of an ambiguous point-light walker. Perception, 33, 547-560. doi:10.1068/p5004

Vanrie, J., \& Verfaillie, K. (2004). Perception of biological motion:
A stimulus set of human point-light actions. Behavior Research Methods, Instruments, \& Computers, 36, 625-629.

Vanrie, J., \& Verfaillie, K. (2006). Perceiving depth in point-light actions. Perception \& Psychophysics, 68, 601-612.

Vanrie, J., \& Verfaillie, K. (2008). Perceptual coupling of multiple point-light figures. Visual Cognition, 16, 585-615. doi:10.1080/ 13506280701269334

WichmanN, F. A., \& Hill, N. J. (2001a). The psychometric function: I. Fitting, sampling, and goodness of fit. Perception \& Psychophysics, 63, 1293-1313.

WichmanN, F. A., \& HiLl, N. J. (2001b). The psychometric function: II. Bootstrap-based confidence intervals and sampling. Perception \& Psychophysics, 63, 1314-1329.

(Manuscript received November 23, 2009: revision accepted for publication February 26, 2010.) 